\title{
Modeling Forced Flow Chemical Vapor Infiltration Fabrication of SiC-SiC Composites for Advanced Nuclear Reactors
}

\author{
Christian P. Deck, H. E. Khalifa, B. Sammuli, and C. A. Back \\ General Atomics, P.O. Box 85608, San Diego, CA 92186-5608, USA \\ Correspondence should be addressed to Christian P. Deck; christian.deck@ga.com
}

Received 21 November 2012; Accepted 5 February 2013

Academic Editor: Hangbok Choi

Copyright (c) 2013 Christian P. Deck et al. This is an open access article distributed under the Creative Commons Attribution License, which permits unrestricted use, distribution, and reproduction in any medium, provided the original work is properly cited.

\begin{abstract}
Silicon carbide fiber/silicon carbide matrix ( $\mathrm{SiC}-\mathrm{SiC}$ ) composites exhibit remarkable material properties, including high temperature strength and stability under irradiation. These qualities have made $\mathrm{SiC}$-SiC composites extremely desirable for use in advanced nuclear reactor concepts, where higher operating temperatures and longer lives require performance improvements over conventional metal alloys. However, fabrication efficiency advances need to be achieved. SiC composites are typically produced using chemical vapor infiltration (CVI), where gas phase precursors flow into the fiber preform and react to form a solid SiC matrix. Forced flow CVI utilizes a pressure gradient to more effectively transport reactants into the composite, reducing fabrication time. The fabrication parameters must be well understood to ensure that the resulting composite has a high density and good performance. To help optimize this process, a computer model was developed. This model simulates the transport of the SiC precursors, the deposition of $\mathrm{SiC}$ matrix on the fiber surfaces, and the effects of byproducts on the process. Critical process parameters, such as the temperature and reactant concentration, were simulated to identify infiltration conditions which maximize composite density while minimizing the fabrication time.
\end{abstract}

\section{Introduction}

Advanced nuclear reactor concepts promise significant improvements over current technology, including increased efficiency, higher fuel burn-up, and longer core life. However, these features put increasing demands on the performance of fuel cladding and other reactor components, and materials must be developed for these reactors that are both resistant to high levels of irradiation damage and offer accident tolerant behavior. Silicon carbide fiber/silicon carbide matrix ( $\mathrm{SiC}-\mathrm{SiC})$ composites offer many desirable properties, and are being considered for use in advanced nuclear reactor designs, such as the General Atomics Energy Multiplier Module $\left(\mathrm{EM}^{2}\right)$ concept. Experiments on monolithic silicon carbide have shown that it maintains excellent mechanical performance in harsh, high temperature, and high irradiation rate environments, but its low toughness limits its application [1-3]. High purity and high quality SiC fiber-reinforced composites have shown similar performance under harsh conditions but offer improved toughness to address this limitation. In these composites, a silicon carbide matrix is deposited within a preform composed of high purity, nearstoichiometric silicon carbide fibers, such as Tyranno-SA fibers (Ube Industries, Ube, Japan) or Hi-Nicalon type $\mathrm{S}$ fibers (Nippon Carbon Co., Ltd., Tokyo, Japan). The performance of these composites has the potential to enable the development and construction of high temperature, long-life advanced reactor concepts.

Several techniques have been developed to fabricate $\mathrm{SiC}$ matrix composite materials, including melt infiltration, polymer infiltration and pyrolysis, and chemical vapor infiltration (CVI) [4-6]. However, in order to achieve good irradiation resistance, very high purity material is required, and CVI is the most reliable approach to produce a sufficiently pure matrix for nuclear applications. In CVI, a silicon carbide precursor (or precursors) is introduced into a high temperature chamber in the gas phase. This is commonly done under vacuum, and the precursors are allowed to diffuse 
into the preform and chemically react, forming a silicon carbide matrix within the sample. The most commonly used precursor is methyltrichlorosilane $\left(\mathrm{CH}_{3} \mathrm{SiCl}_{3}\right)$, which is mixed with hydrogen and decomposes to form silicon carbide according to (1) [1]

$$
\mathrm{CH}_{3} \mathrm{SiCl}_{3(\mathrm{~g})}+\mathrm{H}_{2(\mathrm{~g})} \longrightarrow \mathrm{SiC}_{(\mathrm{s})}+3 \mathrm{HCl}_{(\mathrm{g})}+\mathrm{H}_{2(\mathrm{~g})} .
$$

While CVI produces a very high purity matrix, the deposition process is dependent on the diffusion of reactants into the fiber preform, and a slow reaction rate can be desirable to ensure uniform transport of reactants throughout the fiber preform. The reaction rate can be controlled through selection of the deposition parameters; for example, higher process temperatures generally lead to more rapid reaction and higher deposition rates. Uniform matrix deposition is essential to achieve high composite density and good material properties, but by slowing the reaction rate, the fabrication process may require long infiltration times. For fiber-reinforced composite materials, small voids between fibers within the tows are vulnerable to being closed off by matrix deposits on the tow surface. Because of this, density uniformity is especially important for thicker samples, and reduced density can lead to reduced material performance. Often, a density gradient will exist in the composite, where the densest regions are located near the surface (where the precursors first reach the composite), and the least dense areas are located towards the center, especially inside fiber tows. A schematic of this conventional CVI is shown in Figure 1(a).

Several approaches to reduce fabrication time have been reported in the literature. Two of the more promising routes are thermal gradient chemical vapor infiltration and pressure gradient chemical vapor infiltration (also called forced flow CVI). In thermal gradient CVI, modifications to both the CVI chamber and fixtures are designed to establish a temperature gradient in the opposite direction of the diffusionrelated reactant concentration gradient. This allows for faster reaction rates near the center of the composite, which helps offset the reduction in precursor concentration, and reduces the overall infiltration time needed to make the composite. In forced flow CVI (FFCVI), a pressure gradient is established to enhance reactant transport into the fiber preform (compared to transport via diffusion only). By improving transport of reactants into the preform, the depletion of the precursors is reduced, and conditions allowing for faster deposition rates can be used while still achieving acceptable final composite densities. However, this approach must be carefully controlled, as variations in pressure in different regions of the composite can have detrimental effects on the matrix infiltration uniformity. In addition, it is necessary to hold the sample in more complicated fixtures to direct the flow of reactants (Figure 1(b)).

In order to help understand the effects of different parameters on the composite infiltration process, the diffusion and chemical reaction of the precursors can be modeled. Several approaches have been reported in the literature, including modeling chemical compositions of the gas and solid phases [7], modeling diffusion through a fibrous preform [8], and simulating precursor concentrations on a sample in a typical reactor [9]. These models can be used to consider the effects of different reaction parameters, although the trends predicted by some models have not always been consistent with experimentally observed results, and simplified models cannot account for all the phenomena occurring during deposition.

In this work, $\mathrm{SiC}-\mathrm{SiC}$ composites were fabricated by chemical vapor infiltration, and an empirical model was developed to simulate forced-flow CVI. This work expands on a model which we had previously developed [10] to simulate the infiltration process at the fiber scale $(\sim 10-100 \mu \mathrm{m})$. In these current results, the effects of forced flow are included in the simulation. The infiltration simulated by the model was qualitatively similar to that observed in the experiments, and the effects of different fabrication parameters were investigated.

\section{Sample Fabrication and Characterization}

$\mathrm{SiC}$ matrix composites were fabricated under a range of conditions, including forced flow CVI and conventional CVI (with no pressure gradient). Various composite geometries can be fabricated; however, for most of this work, the material was produced as larger planar sheets that were then cut into samples appropriate for different characterization techniques.

The sample fabrication process has been described previously [10]. Briefly, $\mathrm{SiC}$ fiber fabric is cut to shape and stacked to achieve a nominally $1 \mathrm{~mm}$ thick preform of $\sim 35 \%$ fiber volume fraction. This fiber preform is then processed under vacuum and at elevated temperatures $\left(900-1100^{\circ} \mathrm{C}\right)$ to form a composite using chemical vapor infiltration. This infiltration process is used to first deposit a thin pyrolytic carbon interface layer over the fibers and then infiltrate the $\mathrm{SiC}$ matrix. The silicon carbide matrix was deposited from a dilute reactant mixture composed of methyltrichlorosilane (MTS) evaporated into a hydrogen flow.

Samples were infiltrated using both conventional (isobaric) CVI and forced flow CVI, and all samples were weighed prior to infiltration and at regular intervals during infiltration to monitor weight gain as a function of processing time. Forced flow samples were processed until the pressure differential through the sample increased approximately tenfold from the starting pressure drop, which varied with the fiber preform size and structure. Depending on the deposition conditions, this process took up to 3 days. In order to allow a more direct comparison, the durations of the conventional CVI runs were selected to yield samples with similar densities to the forced flow samples, and these runs took significantly longer than the forced flow runs. The relative sample density was calculated from the measured sample volume and mass and was compared to the maximum theoretical density of $3.2 \mathrm{~g} / \mathrm{cm}^{3}$ for SiC fiber-reinforced samples.

\section{Modeling SiC Matrix Infiltration}

In order to help optimize infiltration process parameters, a two-dimensional computer simulation was developed to 


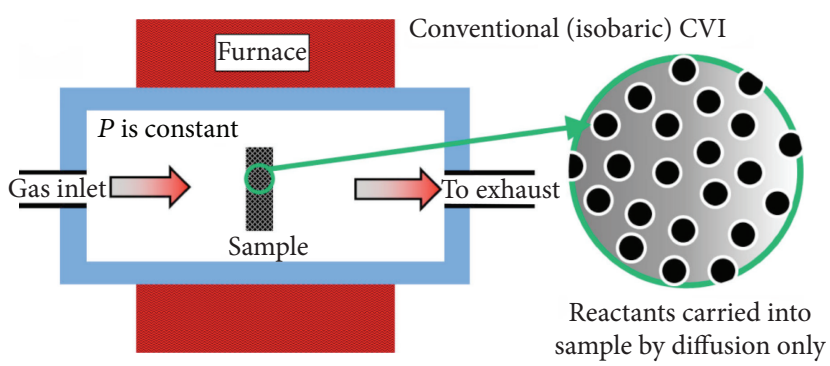

(a)



(b)

FIgURE 1: (a) Schematic of conventional (isobaric) CVI and (b) schematic of forced flow or pressure gradient CVI.

model the effects of different deposition conditions on the infiltration process. To accurately simulate the matrix infiltration process, the transport of the reactants through the fiber preform must be followed. However, due to the size of the fibers (7-10 $\mu \mathrm{m}$ diameter), a system including several fibers would be much too large (approximately $100 \mu \mathrm{m}$ ) to practically model and track the motions of individual reactant molecules. Typical Knudsen numbers $(\mathrm{Kn}=$ mean free path $\lambda$, divided by characteristic length $L$ ) for deposition conditions are also too large $(\lambda / L \gg 1)$ to use standard computational fluid flow codes to model the infiltration. In order to address these restrictions and accommodate the larger system size, the model uses a direct simulation Monte Carlo (DSMC) solver with the OpenFOAM toolbox.

With the DSMC approach, statistically representative parcels are tracked, rather than individual molecules, and each parcel contains many particles. During the simulation of conventional (isobaric) infiltration, the initial velocity of each parcel is obtained from a thermal velocity distribution function. In the simulation of forced flow infiltration, a net velocity is applied to the parcels in the model, with this velocity representing the effect of a pressure gradient on the reactant gas flow. In both cases, the subsequent motions of the parcels are tracked ballistically, accounting for collisions with walls and other particles.

In addition to tracking the motion of different parcels, the DSMC solver also tracks the reaction of MTS on surfaces to produce $\mathrm{SiC}$. The general growth rate equation used in the model is $G=S_{\mathrm{MTS}} F_{\mathrm{MTS}}$, where $G$ is the growth rate, $S_{\text {MTS }}$ is the sticking coefficient, or probability that MTS will react to form silicon carbide on a surface, and $F_{\mathrm{MTS}}$ is the flux of MTS on that surface. The sticking coefficient of MTS is given by $S_{\mathrm{MTS}}^{0}=A \exp \left(-E_{a \mathrm{MTS}} / R T\right) \sqrt{P_{\mathrm{H}_{2}}}$, where $P_{\mathrm{H}_{2}}$ is the partial pressure of hydrogen in the system, $E_{a \mathrm{MTS}}$ is the activation energy for MTS decomposition, $R$ is the natural gas constant, $T$ is the temperature, and $A$ is a constant [11]. This $\mathrm{SiC}$ deposition is modeled by depleting the number of MTS particles in each parcel in contact with a surface. The simulation also accounts for the presence of $\mathrm{HCl}$ byproducts, which have been shown both experimentally and in other models to inhibit the deposition process [11-14]. $\mathrm{HCl}$ can occupy surface sites, and the probability of MTS conversion to silicon carbide is reduced by $1-\theta / \theta_{m}$, in proportion to the surface area covered by adsorbed $\mathrm{HCl}\left(\theta / \theta_{m}\right)$.
As MTS decomposes on the surface to form SiC, $\gamma$ is the fraction of $\mathrm{HCl}$ that remains on the surface, and the $\mathrm{HCl}$ adsorption rate is given by (2), where $S_{\mathrm{HCl}}^{0}$ is the sticking coefficient of $\mathrm{HCl}$ on the surface and $F_{\mathrm{HCl}}$ is the flux of $\mathrm{HCl}$ particles on the surface:

$$
\frac{d \theta}{d t}=\left(S_{\mathrm{MTS}}^{0} \gamma F_{\mathrm{MTS}}+S_{\mathrm{HCl}}^{0} F_{\mathrm{HCl}}\right)\left(1-\frac{\theta}{\theta_{m}}\right) .
$$

$\mathrm{HCl}$ will also desorb from the surface according to $d \theta / d t=$ $-\nu \theta \exp \left(-E_{a \mathrm{HCl}} / R T\right)$, where $E_{a \mathrm{HCl}}$ is the activation energy for the desorption of $\mathrm{HCl}$ and $v$ is a rate constant. The steady state growth rate is given by (3), where $C$ is a constant:

$$
G=\frac{S_{\mathrm{MTS}}^{0} F_{\mathrm{MTS}} C}{1+\left(\gamma S_{\mathrm{MTS}}^{0} F_{\mathrm{MTS}}+S_{\mathrm{HCl}}^{0} F_{\mathrm{HCl}}\right) / \theta_{m} \nu \exp \left(-E_{a \mathrm{HCl}} / R T\right)} .
$$

In this equation, the activation energies are taken from the literature, with $E_{a \mathrm{MTS}}=188 \mathrm{~kJ} / \mathrm{mol}$ [12] and $E_{a \mathrm{HCl}}=$ $268 \mathrm{~kJ} / \mathrm{mol}$ [13]. SiC deposition experiments were performed across a range of different process conditions, and coating rates were measured and compared to the simulation in order to empirically fit values for the MTS and $\mathrm{HCl}$ sticking coefficients and $\gamma$. For each collision between a given parcel and a surface, the modified DSMC solver uses the growth rate equation to determine the amount of MTS that reacts to form $\mathrm{SiC}$. The solver also tracks the $\mathrm{HCl}$ generated by the MTS decomposition, of which a fraction remains on the surface (given by $\gamma$ ), and the remainder is added to the parcel. The solver represents the deposited $\mathrm{SiC}$ by adding elements to fiber surfaces upon which $\mathrm{SiC}$ has been deposited, increasing the fiber diameter and reducing the open pore volume.

\section{Results and Discussion}

4.1. Forced Flow Chemical Vapor Infiltration. Forced flow chemical vapor infiltration offers a means to increase transport of reactants through the fiber preform during the matrix infiltration process. As a result, process conditions that allow for more rapid deposition can be used, such as higher temperatures and MTS partial pressures. This can reduce process time, while the pressure gradient helps ensure matrix deposition uniformity. 


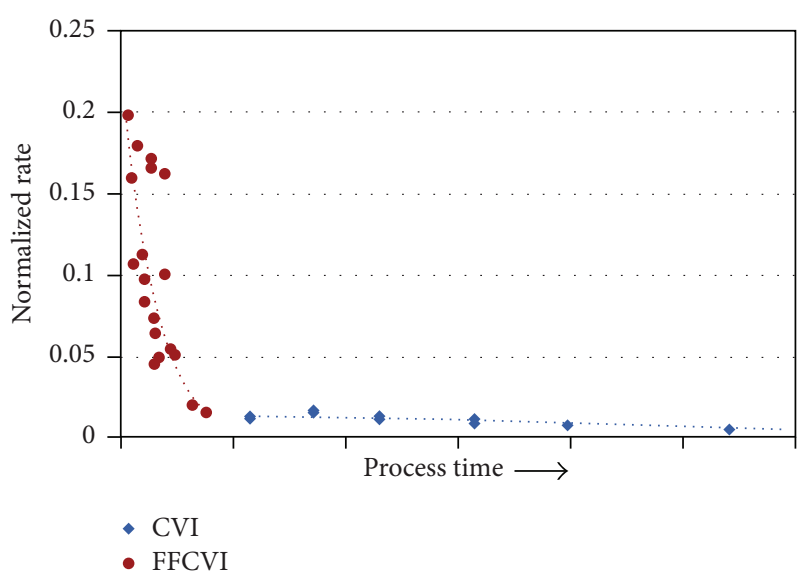

(a)

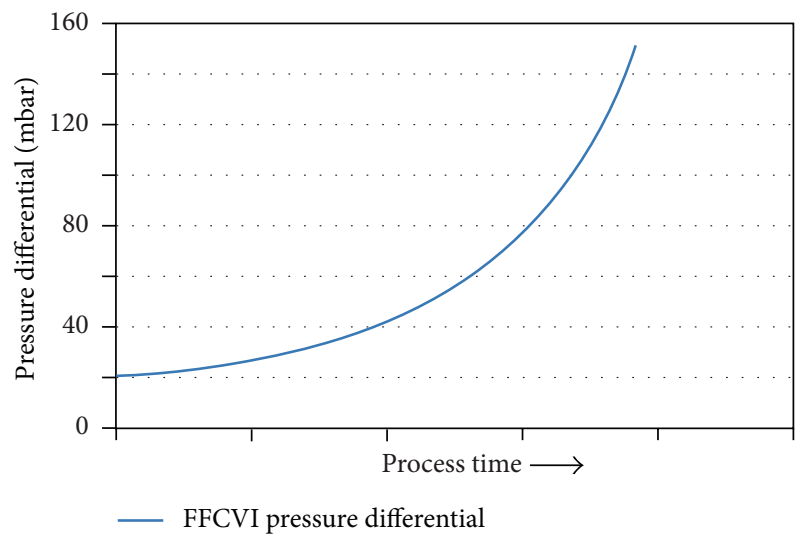

(b)

Figure 2: (a) Plot of normalized rate of mass gain as a function of process time for conventional CVI and FFCVI and (b) plot of increasing pressure differential across sample observed during FFCVI.

The infiltration process was interrupted at several intervals in order to measure the mass gain of the samples and remove portions for characterization. It was found that the infiltration process could be started and stopped in this manner without causing discontinuities in the matrix. Figure 2(a) shows a typical rate of mass gain for conventional CVI and forced flow CVI. Also plotted is the pressure differential between the inlet and exhaust sides of the sample in the forced flow case (Figure 2(b)). As a result of the improved reactant transport into the sample during FFCVI, higher deposition rates can be used while still achieving reasonable infiltration uniformity.

For both FFCVI and isobaric CVI, the rate of mass gain typically starts out high, and then drops as the infiltration process continues. The amount of $\mathrm{SiC}$ deposited on the samples is a function of CVI conditions but also of the available surface area of the substrate. Initially, the fiber preform has a very high surface area, and consequently, a high rate of mass gain is observed. However, as the matrix fills in the voids within the preform, some regions become closed off, trapping voids within the sample. This reduces the available surface area as well as the rate of mass gain.
In conventional CVI, very low deposition rates are used to allow sufficient reactant diffusion into the preform. The added pressure gradient in FFCVI allows for higher deposition rates to be achieved while maintaining uniformity through the sample. If process conditions are changed in a conventional CVI process to achieve deposition rates comparable to FFCVI, a significant $\mathrm{SiC}$ deposition gradient develops from the surface of the sample (the surface exposed to the reactant flow) towards the center of the sample (Figure 3(a)). The gradient is much reduced when a forced flow configuration is used to achieve similar deposition rates (Figures 3(b) and 3(c)).

In both conventional and forced flow CVI, the rate of mass gain drops with time, as pores are closed off and the available surface area for deposition is reduced. In conventional CVI, the reactants are flowing around the sample, so $\mathrm{SiC}$ deposition on the sample and this eventual pore closure do not affect the process pressure. However, in forced flow CVI, all reactants are forced to flow through the sample, and as the open porosity begins to be filled or closed off, the pathways for gas flow are reduced, and the pressure differential across the samples increases. This pressure difference increases rapidly towards the end of the process and can result in the upstream side of the sample seeing significantly higher pressures than the exhaust. As the deposition rate can be strongly dependent on reactant partial pressures, this increasing pressure differential can lead to nonuniform deposition (Figure 4). This can result in excess $\mathrm{SiC}$ build-up on the inlet side of the composite and can also result in deposition conditions that produce nonstoichiometric, silicon-rich SiC.

4.2. Simulation of SiC Matrix Deposition. Although forced flow CVI can be used to potentially provide increased deposition rates, the conditions must be understood to ensure uniform infiltration. To more rapidly investigate the effects of various process parameters and sample geometries on the fabrication process, a computer model was used to simulate $\mathrm{SiC}$ infiltration into an idealized fiber arrangement. The influence of different process parameters was explored in the context of the maximum simulated composite density achieved and the infiltration duration required to reach that density. The end of the simulation was defined as the time at which the reactant transport pathways to the inside fibers became completely blocked off; after this point, any additional deposition would occur only on the surface, and the composite density and pore volume would no longer change.

Prior to the start of infiltration, the structure of the composite consists of bundles or tows of hundreds or thousands of $\mathrm{SiC}$ fibers, and these tows are woven into the desired macroscopic component geometry. The simulation developed in this work focuses on the deposition at the microscale, and the geometry and boundary conditions were selected to provide an approximation of an actual fiber bundle. Although transport of reactants within the CVD chamber and between adjacent tows (on the macroscopic scale) is also important to the overall composite uniformity, running the simulation 


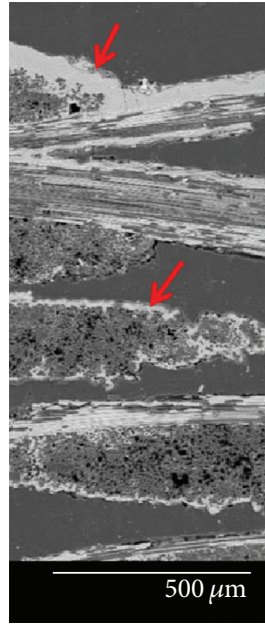

(a)

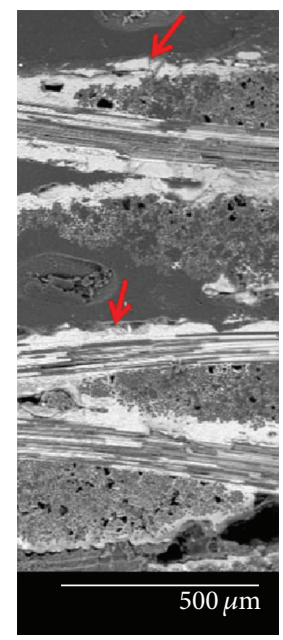

(b)

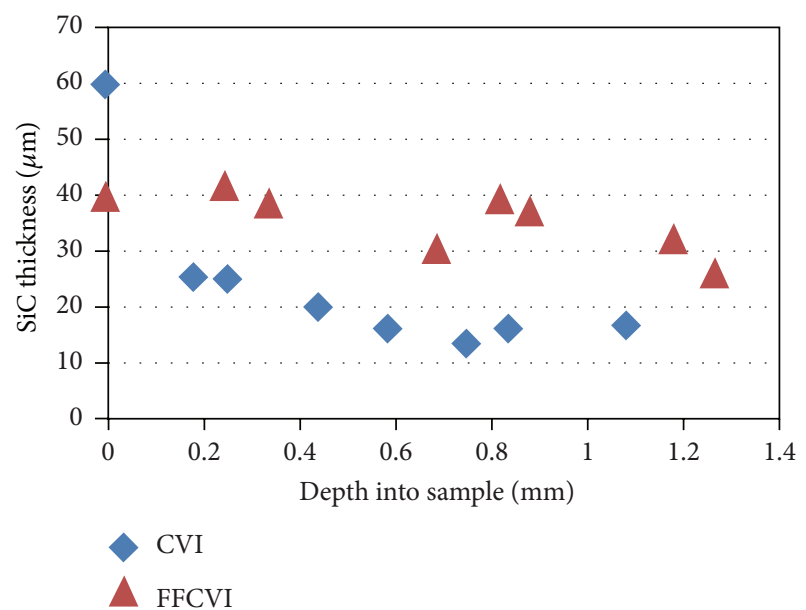

(c)

FiguRE 3: (a) Cross-section of fiber preform partially infiltrated with higher rate isobaric CVI, (b) cross-section of fiber preform partially infiltrated with forced flow CVI, and (c) SiC deposit thickness as a function of depth into the sample for CVI and FFCVI.

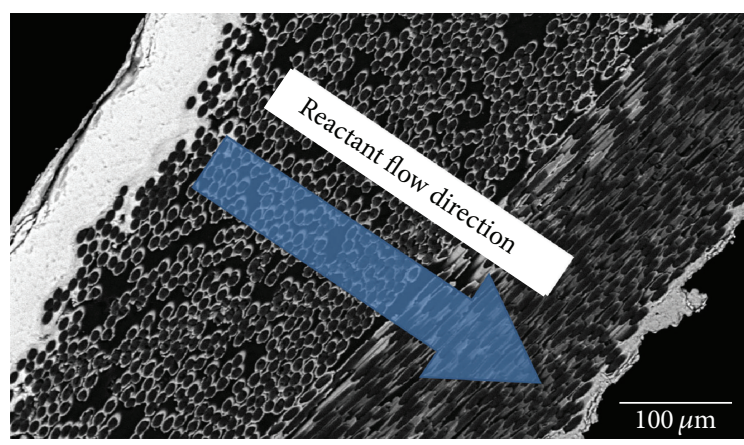

Figure 4: SiC build-up on the upstream side of the sample caused by increasing reactant pressure and varying deposition conditions on the upstream side of the sample during FFCVI.

at this scale would be too computationally intensive to be practical.

Infiltration into the fiber tows was simulated with a narrow column of fiber cross-sections. Fiber spacing was kept constant, although the simulation approach could also be used to model a more realistic system or randomly arranged fibers. The fiber tows used in these composites have elliptical cross-sections, and the column of fibers used in the simulation represents approximately half the yarn minor axis, as shown in Figure 5. Reactant gases were introduced from one side, and a net velocity could be applied to the reactants to simulate forced flow conditions. The boundary conditions used for the other sides of the system are shown in Figure 5(c).

With the empirically determined reactant sticking coefficients, the simulated $\mathrm{SiC}$ infiltration is qualitatively similar to the experimentally observed deposition gradient, as shown in Figure 5(d). For this comparison, the coating thickness was measured as a function of position within the fiber bundle, and the model reproduced the variation in coating thickness away from the tow surface that was observed in the actual sample. The simulation was then used to model the infiltration for both isobaric and forced flow conditions and to investigate the effects of different coating parameters on the deposition process. Certain parameters (including temperature, pressure, and MTS concentration) were varied within ranges that coincided with the typical conditions used in the experiments.

4.2.1. Reactant Velocity Effects. In the model, reactants can be introduced with a net velocity which can be set for different forced flow conditions or reduced to zero to represent isobaric (conventional) CVI. At very low velocities, reactant transport into the fiber preform occurs due to diffusion. If this transport is slow relative to the reaction rate of the precursors, $\mathrm{SiC}$ will be deposited towards the outer surface of the sample, and a large deposition gradient will lead to a composite with low final density and high internal porosity. Slow deposition rates are needed to achieve higher final composite densities.

If the reactants have a positive net velocity, this provides additional means to facilitate their transport into the fiber preform. At moderate velocities, the time scale for transport through the sample is comparable to the reaction rate of the precursors, and deposition uniformity is improved. This allows a faster reaction rate to be used which reduces composite fabrication time while maintaining improved uniformity. However, the simulation indicated that if the reactant velocities are very high, transport through the fiber preform can be too fast, and the short residence time can reduce the deposition uniformity. This effect could be exaggerated in our simulation, as the system volume is very small relative to an actual sample (which would be thicker and contain many thousands of fibers). Thus, a given velocity in the model could be sufficient to transport the precursors out of the system before they can completely react, whereas in an actual sample, these unreacted precursors would react and deposit elsewhere. 


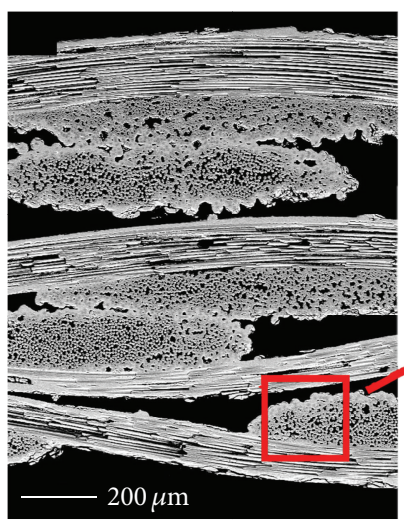

(a)

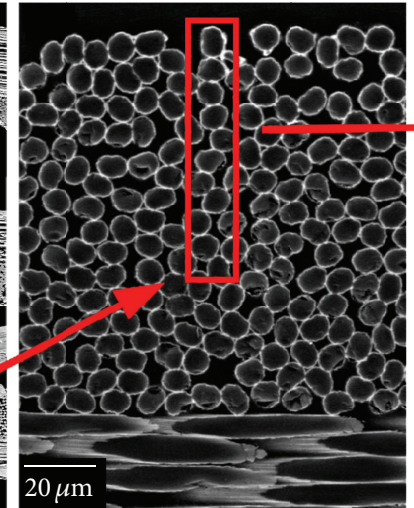

(b)

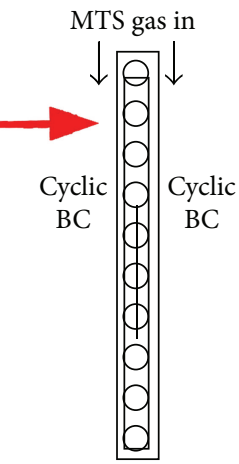

Symmetry plane

(c)

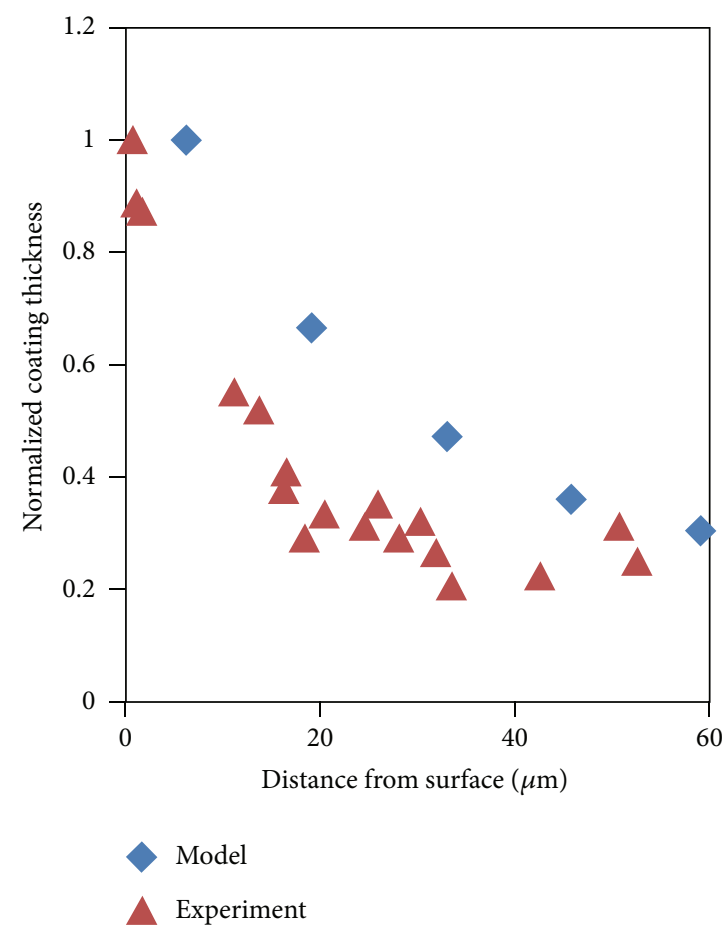

(d)

Figure 5: (a) Cross-section of stacked fabric layers, (b) cross-section of fiber bundle, (c) model geometry showing MTS introduction and boundary conditions, and (d) comparison between simulated and experimentally observed SiC deposition gradients into the fiber preform.

To examine the influence of reactant velocity, the model was run with zero, moderate, and fast reactant velocities. The precursor partial pressure was held constant, and infiltration was simulated at two different $\mathrm{SiC}$ deposition temperatures $\left(900^{\circ} \mathrm{C}\right.$ and $\left.1100^{\circ} \mathrm{C}\right)$. These temperatures are in the range of infiltration temperatures commonly reported in the literature [5]. Reactant velocity was found to have a significant effect on the deposition uniformity and the corresponding final composite relative density. For both slow and moderate reactant velocities, similar deposition was seen at the surface. However, the improved reactant transport achieved by forced flow CVI was apparent in the simulated deposition inside the fiber preform. Moderate velocities (which could be obtained under appropriate FFCVI conditions) provided the highest relative density and most uniform infiltration, as shown in Figure 6(a). The effects of reactant velocity were similar for both temperatures modeled (Figure 6(b)).

4.2.2. Reactant Concentration Effects. The reactant partial pressure also plays an important role in the infiltration process. The MTS flux at the fiber surface is proportional to the MTS partial pressure, and the growth rate increases with the increasing flux (according to (3)). In the model, the reactant concentration is varied by setting the MTS partial pressure (a function of the MTS concentration and the overall pressure). Unlike isobaric infiltration, in FFCVI, the overall pressure cannot be simply controlled. Typically, the downstream pressure is fixed, but unless a bypass is used, 


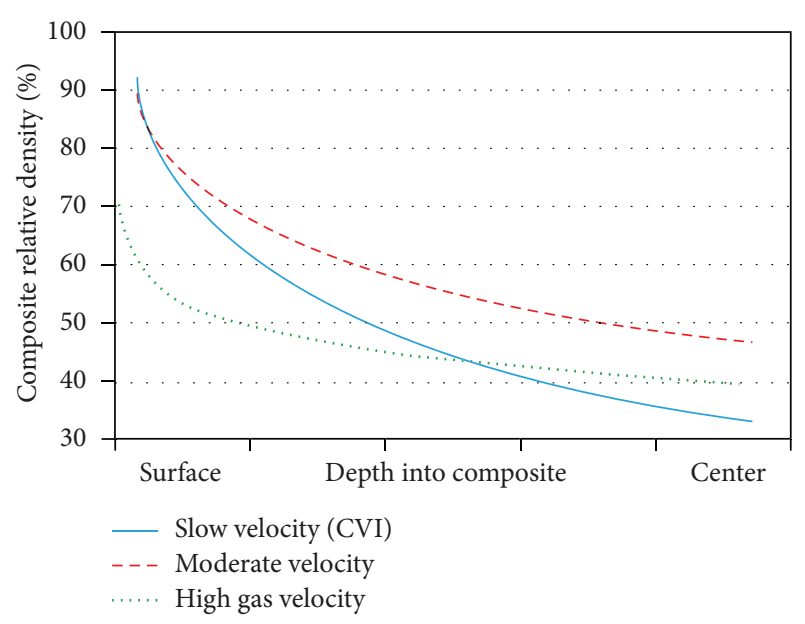

(a)

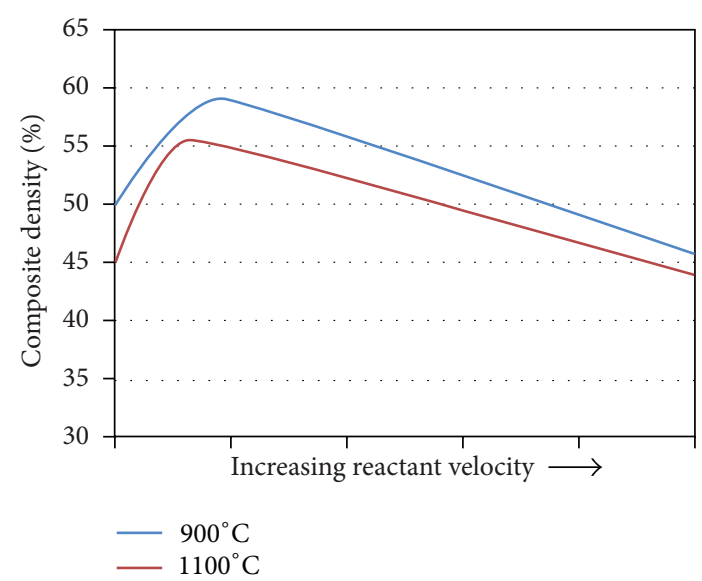

(b)

FIGURE 6: (a) Simulated deposition gradient for varying reactant velocities and (b) variation in simulated composite density as a function of temperature and reactant velocity.

the upstream pressure increases as the transport pathways through the fiber preform close during infiltration. As the process pressure then varies as a function of time and position within the sample, control over the reactant concentration becomes the means by which the reactant partial pressure can be set.

Simulations and experiments were carried out over a range of MTS concentrations, with temperature and reactant velocity held constant. For low MTS concentrations, SiC deposition can be limited by reactant availability. With increasing reactant concentration, the reaction becomes limited by surface site availability, and the deposition rate is no longer strongly influenced by reactant concentration. Both the simulation and experimental results show this effect, and the infiltration process becomes less sensitive to the MTS partial pressure at high MTS concentrations. The simulated infiltration as a function of depth into the composite is shown in Figure 7(a), and for moderate and higher MTS concentrations, there is a minimal effect on the infiltration.
Model results were also compared with experimental results for a range of MTS partial pressures (Figure 7(c)). These experiments were carried out in both CVI and FFCVI conditions, and the infiltration duration was limited (which limited the densities that could be achieved). At higher MTS partial pressures, there was good agreement between the simulated and experimental densities, but at lower partial pressures, the experimental densities were much lower than the simulation. This is likely caused by reactant depletion in the experiment before the precursors reach the fiber preform. Precursors can react on other surfaces inside the furnace, and with very low reactant partial pressures, this can deplete the reactant concentration enough to impact the sample density achieved. At higher reactant partial pressures, the relative amount of depletion is greatly reduced, and in the simulation, the reactants are introduced directly at the fiber preform surface, so the depletion in other regions of the furnace is not considered.

4.2.3. Fiber Spacing Effects. The final parameter modeled in this work is the average fiber spacing in the preform. This variable is governed by the fiber architecture and sample preparation and is unlike reactant pressure or velocity, in that it cannot be set during the actual infiltration. However, the same simulation can be used to model the effects of different fiber spacing by increasing the system size. Coupled with the cyclic boundary conditions, this effectively increases the width of the gaps between adjacent fibers, which allows for more infiltration to occur before the reactant pathways become blocked.

In this study, the width of the simulated system was increased from 15 to $30 \mu \mathrm{m}$. This effectively tripled the gap between adjacent fibers from a $7.5 \mu \mathrm{m}$ gap to a $22.5 \mu \mathrm{m}$ gap. More $\mathrm{SiC}$ deposition is required before these larger reactant transport pathways are closed off. As a result, prolonged infiltration is required for this closure to occur, and a 3.1x increase in processing time was observed in the simulation. This is roughly proportional to the $3 \mathrm{x}$ increase in gap width used in this study. While increased fabrication time is not desirable in terms of rapid processing, if this is accompanied by an increase in composite density and performance, then these gains could offset the long infiltration required. At the end of infiltration, the simulated composite with the larger gap spacing showed an increase of $57 \%$ and $39 \%$ in density compared to the narrow gap model for the $900^{\circ} \mathrm{C}$ and $1100^{\circ} \mathrm{C}$ simulations, respectively (Figure 8).

Composite density has been shown to have a very significant effect on mechanical and thermal performance, and careful control of the average fiber spacing within the composite could be a means to optimize the tradeoff between fabrication time and improved material properties. However, in increasing the average gap between fibers, the overall fiber volume fraction of fibers in the composite is reduced as well. Maintaining a sufficient fiber loading in the composite is essential to obtain increased fracture toughness and more graceful failure characteristic of a composite, so any adjustments to the fiber architecture would need to be evaluated in the context of composite behavior as well. 


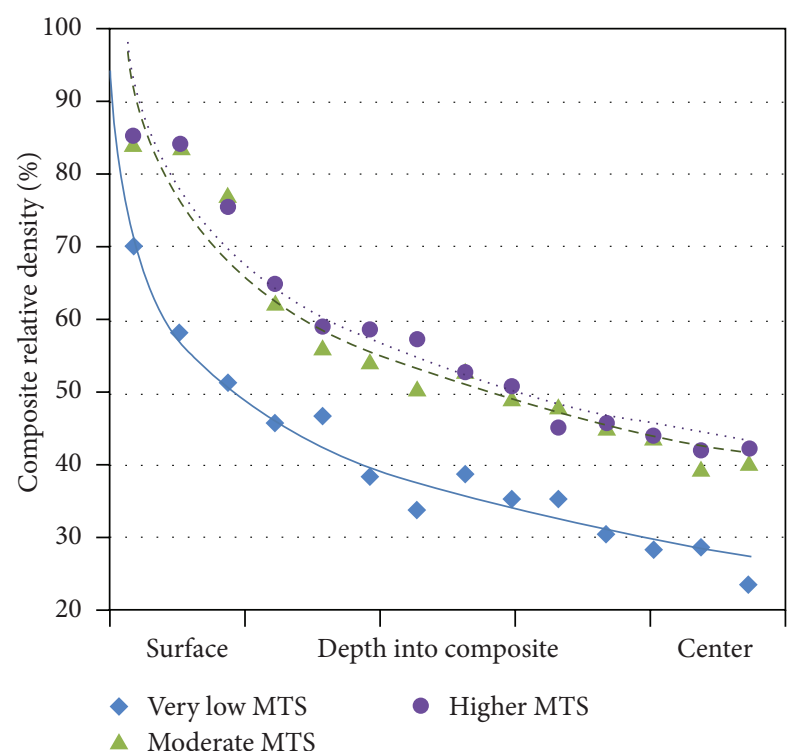

A Moderate MTS

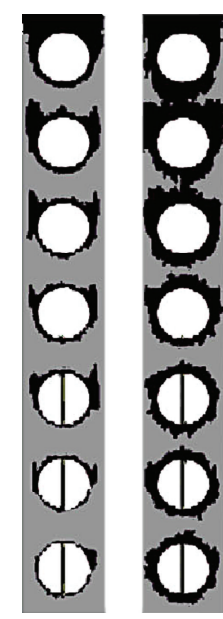

Low MTS High MTS pressure pressure (a)

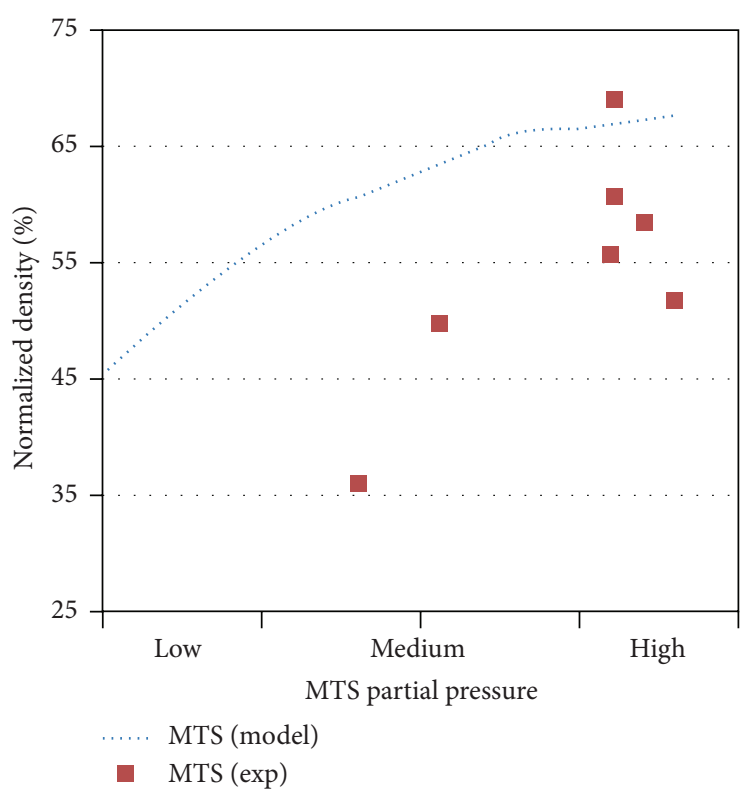

(b)

(c)

FIGURE 7: (a) Simulated deposition gradient for varying MTS partial pressures, (b) simulated infiltration for low and high MTS partial pressures, where black indicates deposited $\mathrm{SiC}$ matrix, and (c) comparison between simulation and experimental results for MTS concentration effects on density.

Simulations of the $\mathrm{SiC}$ infiltration process have also been performed by other groups and trends that are consistent with the results of this work have been reported. Roman et al. $[15,16]$ developed an extensive model of the reaction and deposition process for forced flow CVI and observed similar trends with increasing precursor velocity through the sample. In that work, reactant introduction was given as a volumetric flow, rather than a net velocity, and this makes it difficult to make an exact comparison with this work. However, in both that model and ours, increasing reactant flow from very low to moderate velocities resulted in reduced processing time and increased density and matrix uniformities (Figure 9).

\section{Conclusions}

SiC-SiC composites exhibit many properties that could make them very desirable for use in advanced nuclear reactor concepts, including strength at high temperatures and exceptional stability under irradiation. However, one of the challenges that must be overcome before these materials are widely adopted is the time-consuming and expensive fabrication process required to produce very high purity material. Forced flow CVI is a modification to the conventional isobaric infiltration process, which allows for increased densification rates while maintaining infiltration 


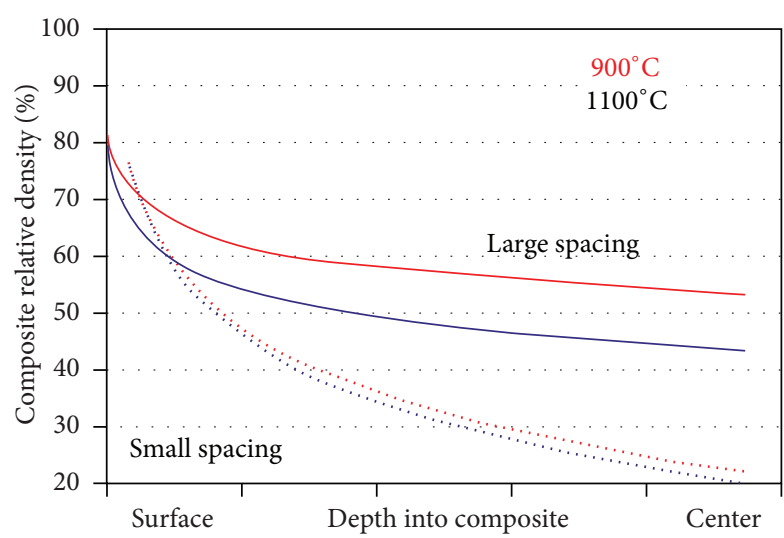

(a)

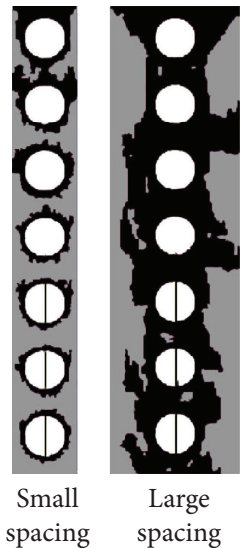

(b)

FIGURE 8: (a) Simulated deposition gradient for varying fiber spacings and temperatures, (b) simulated infiltration for small and large fiber spacing, where black indicates deposited $\mathrm{SiC}$ matrix around white fiber cross-sections.

uniformity. In this work, a model was developed to simulate the chemical vapor infiltration process for the fabrication of $\mathrm{SiC}-\mathrm{SiC}$ composites under both isobaric and forced flow conditions. This model uses a direct simulation Monte Carlo approach and accounts for MTS deposition, depletion, and the inhibition of this reaction due to the $\mathrm{HCl}$ byproducts.

The effects of different fabrication parameters were simulated to investigate ways to optimize forced flow CVI. Increasing reactant flow through the sample increased both the densification rate (reducing the required processing time) and the final composite density. However, very high reactant flow rates were found to reduce uniformity in the simulation, which could be due to insufficient reactant residence time. Very high reactant velocities are also associated with larger pressure gradients across the sample, and in the supporting experimental work, high pressures were found to lead to nonuniform, nonstoichiometric deposition on the sample. The trends of reactant velocity were similar between the model developed in this work and the experimental results and were also consistent with simulations reported in the literature. The concentration of the MTS precursor (and the corresponding partial pressure) was also found to influence the infiltration process, although the effect was most pronounced

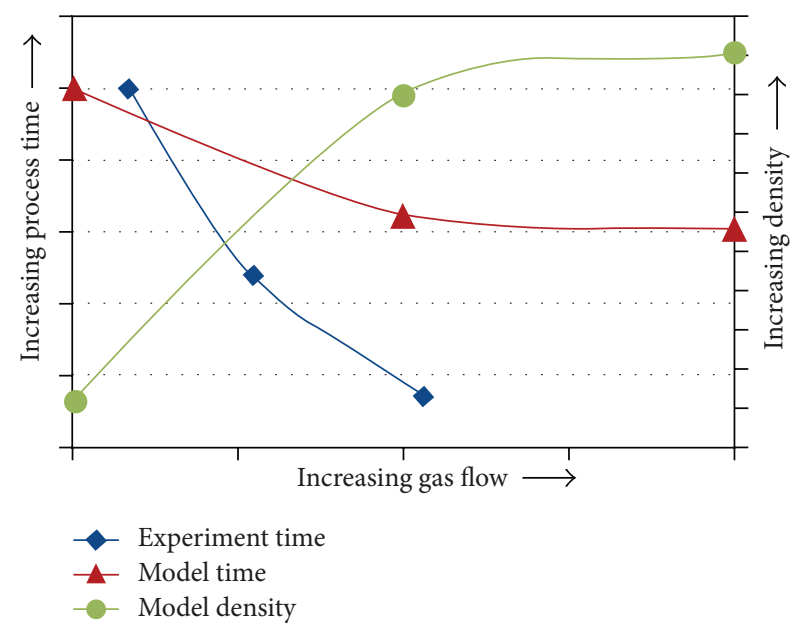

(a)

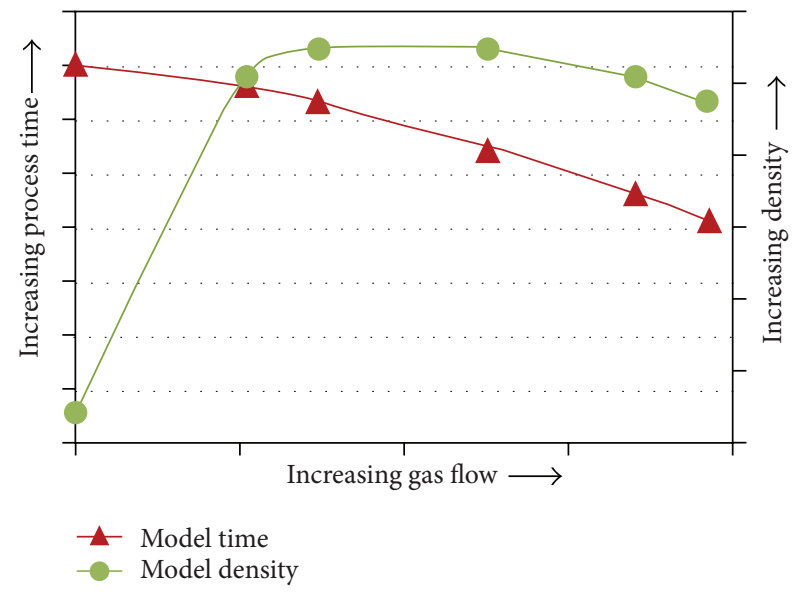

(b)

FIGURE 9: (a) Simulation and experimental results from the current work, showing effects of reactant velocity and (b) simulation results from Roman et al., [15] showing similar trends with increasing gas flow.

at lower concentrations. Finally, an increase in the average spacing between fibers led to a composite with a higher final density, but it increased the required processing time as well. Any infiltration improvements achieved by a modification to the fiber architecture would need to be carefully balanced with any impacts the altered structure had on the mechanical performance of the composite.

Further improvements to the model could allow simulation of reactant transport through larger intertow porosity and also account for randomized fiber positioning and the more complex two- or three-dimensional structure found in woven preforms. The model provides means to explore the effects of different microstructures and infiltration process parameters on the composite densification process. Using this model, these effects can be simulated in far less time than would be required to complete a corresponding experimental test matrix. This allows for a much faster approach towards optimization of the fabrication process, but it needs to be coupled with, and confirmed by, experimental results. More efficient composite fabrication can lead to improved 
consistency and reduced fabrication cost and time, which will bring $\mathrm{SiC}-\mathrm{SiC}$ composites closer to the deployment in actual advanced nuclear reactor applications.

\section{Acknowledgments}

The authors would like to acknowledge the contributions of the $\mathrm{EM}^{2}$ lab personnel to the operation of the $\mathrm{SiC}$ composite fabrication facility at General Atomics. The work presented here was supported by General Atomics internal funding.

\section{References}

[1] L. L. Snead, T. Nozawa, Y. Katoh, T. S. Byun, S. Kondo, and D. A. Petti, "Handbook of $\mathrm{SiC}$ properties for fuel performance modeling," Journal of Nuclear Materials, vol. 371, no. 1-3, pp. 329-377, 2007.

[2] Y. Katoh, T. Nozawa, L. L. Snead, K. Ozawa, and H. Tanigawa, "Stability of $\mathrm{SiC}$ and its composites at high neutron fluence," Journal of Nuclear Materials, vol. 417, no. 1-3, pp. 400-405, 2011.

[3] K. Hironaka, T. Nozawa, T. Hinoki et al., "High-temperature tensile strength of near-stoichiometric $\mathrm{SiC} / \mathrm{SiC}$ composites," Journal of Nuclear Materials, vol. 307-311, no. 2, pp. 1093-1097, 2002.

[4] M. Kotani, A. Kohyama, and Y. Katoh, "Development of SiC/SiC composites by PIP in combination with RS," Journal of Nuclear Materials, vol. 289, no. 1-2, pp. 37-41, 2001.

[5] R. Naslain, "Design, preparation and properties of non-oxide CMCs for application in engines and nuclear reactors: an overview," Composites Science and Technology, vol. 64, no. 2, pp. 155-170, 2004.

[6] K. Shimoda, J. S. Park, T. Hinoki, and A. Kohyama, "Microstructural optimization of high-temperature $\mathrm{SiC} / \mathrm{SiC}$ composites by NITE process," Journal of Nuclear Materials, vol. 386-388, pp. 634-638, 2009.

[7] W. G. Zhang and K. J. Hüttinger, "CVD of SiC from methyltrichlorosilane-part II: composition of the gas phase and the deposit," Chemical Vapor Deposition, vol. 7, no. 4, pp. 173-181, 2001.

[8] G. Y. Chung and B. J. McCoy, "Modeling of chemical vapor infiltration for ceramic composites reinforced with layered, woven fabrics," Journal of the American Ceramic Society, vol. 74, no. 4, pp. 746-751, 1991.

[9] X. Wei, L. Cheng, L. Zhang, Y. Xu, and Q. Zeng, "Numerical simulation of effect of methyltrichlorosilane flux on isothermal chemical vapor infiltration process of $\mathrm{C} / \mathrm{SiC}$ composites," Journal of the American Ceramic Society, vol. 89, no. 9, pp. 27622768, 2006.

[10] C. P. Deck, H. E. Khalifa, B. Sammuli, T. Hilsabeck, and C. A. Back, "Fabrication of SiC-SiC composites for fuel cladding in advanced reactor designs," Progress in Nuclear Energy, vol. 57, pp. 38-45, 2012.

[11] T. M. Besmann, B. W. Sheldon, T. S. Moss III, and M. D. Kaster, "Depletion effects of silicon carbide deposition from methyltrichlorosilane," Journal of the American Ceramic Society, vol. 75, no. 10, pp. 2899-2903, 1992.

[12] C. Lu, L. Cheng, C. Zhao, L. Zhang, and Y. Xu, "Kinetics of chemical vapor deposition of $\mathrm{SiC}$ from methyltrichlorosilane and hydrogen," Applied Surface Science, vol. 255, no. 17, pp. 7495-7499, 2009.
[13] M. T. Schulberg, M. D. Allendorf, and D. A. Outka, "The adsorption of hydrogen chloride on polycrystalline $\beta$-silicon carbide," Surface Science, vol. 341, no. 3, pp. 262-272, 1995.

[14] H. C. Chang, T. F. Morse, and B. W. Sheldon, "Minimizing infiltration times during isothermal chemical vapor infiltration with methyltrichlorosilane," Journal of the American Ceramic Society, vol. 80, no. 7, pp. 1805-1811, 1997.

[15] Y. G. Roman, J. F. A. K. Kotte, and M. H. J. M. de Croon, "Analysis of the isothermal forced flow chemical vapour infiltration process-part I: theoretical aspects," Journal of the European Ceramic Society, vol. 15, pp. 875-886, 1995.

[16] Y. G. Roman, M. H. J. M. de Croon, and R. Metselaar, "Analysis of the isothermal forced flow chemical vapour infiltration process-part II: experimental study," Journal of the European Ceramic Society, vol. 15, no. 9, pp. 887-898, 1995. 


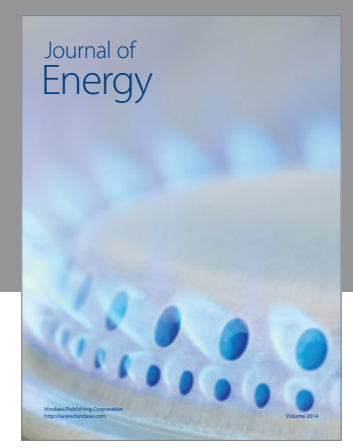

Journal of

Industrial Engineering
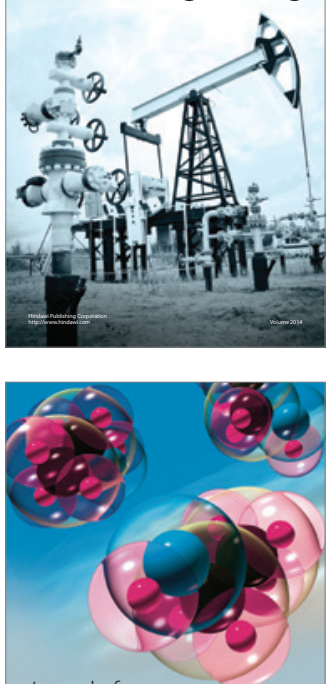

Fuels
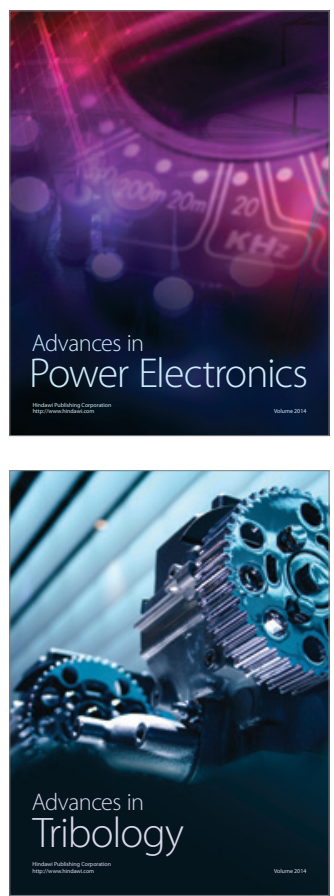

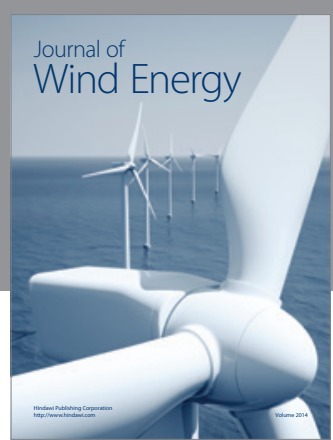

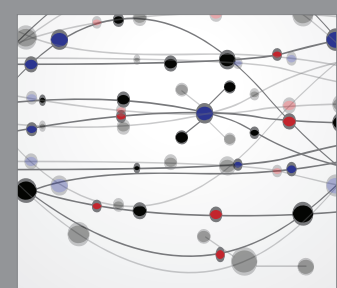

The Scientific World Journal

Submit your manuscripts at http://www.hindawi.com

Journal of

Structures
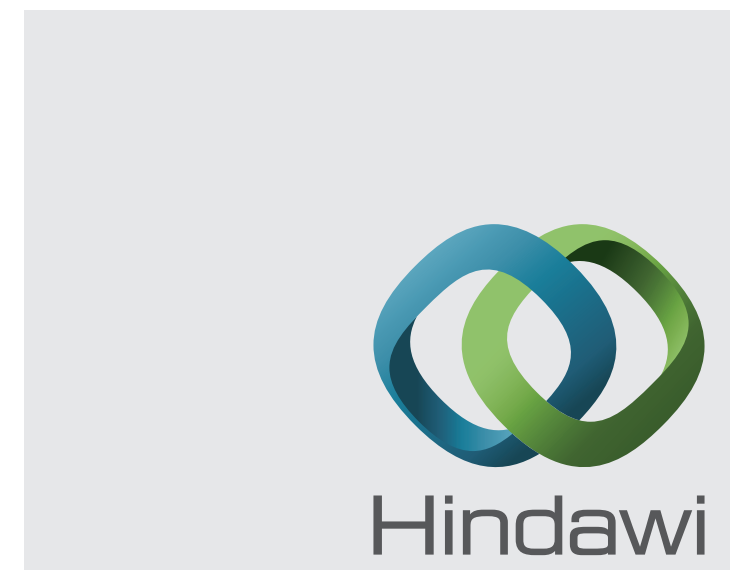

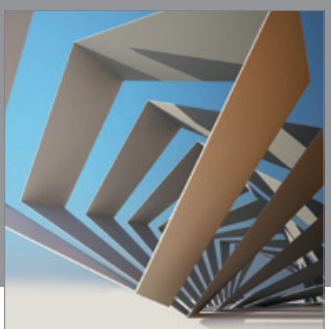

Rotating

Machinery
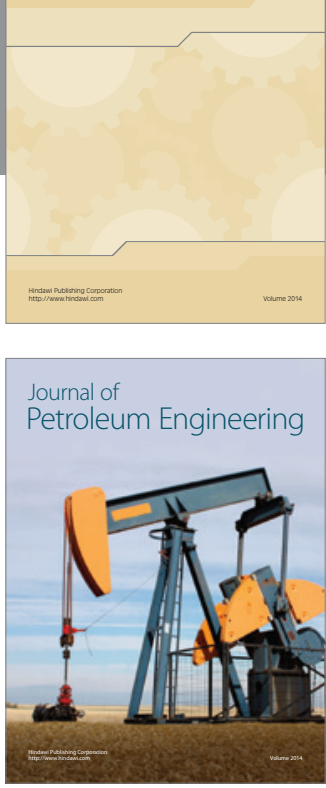

Journal of

Solar Energy
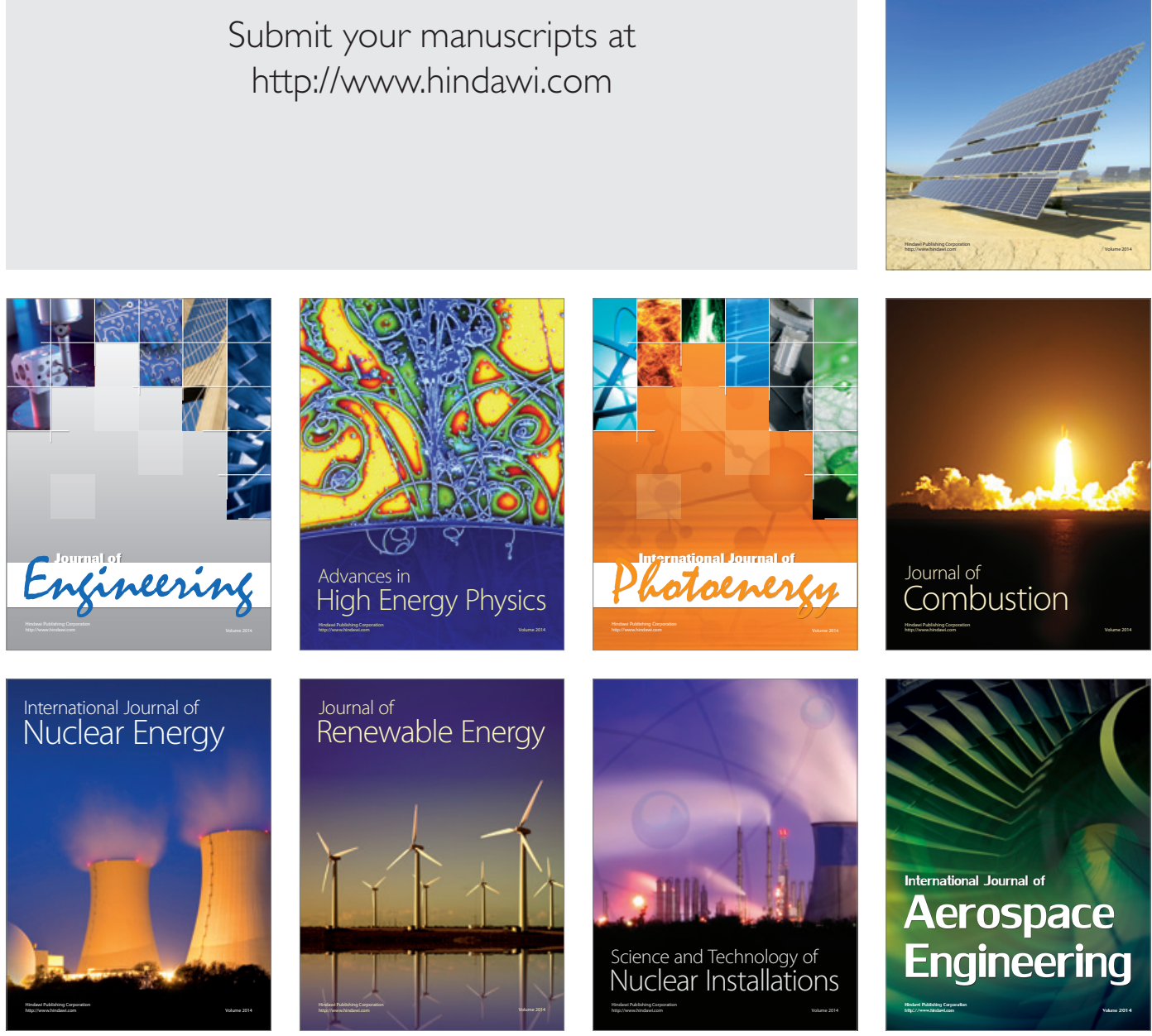